\title{
CAPTURE PURSUIT GAMES ON UNBOUNDED DOMAINS
}

\author{
S. ALEXANDER, R. BISHOP, AND R. GHRIST
}

\begin{abstract}
We introduce simple tools from geometric convexity to analyze capturetype (or "Lion and Man") pursuit problems in unbounded domains. The main result is a necessary and sufficient condition for eventual capture in equal-speed discrete-time multi-pursuer capture games on convex Euclidean domains of arbitrary dimension and shape. This condition is presented in terms of recession sets in unit tangent spheres. The chief difficulties lie in utilizing the boundary of the domain as a constraint on the evader's escape route. We also show that these convex-geometric techniques provide sufficient criteria for pursuit problems in non-convex domains with a convex decomposition.
\end{abstract}

\section{INTRODUCTION}

Games of pursuit and evasion are among the oldest and most elegant problems in game theory, osculating differential equations, control theory, differential geometry, and graph theory. This paper focuses on global geometric features of capture-type pursuit problems. The primary contribution is an introduction of tools from geometric convexity which allow for results so general as to be independent of the number of pursuers, and the dimension and (to a lesser extent) the geometry of the playing field.

1.1. Of lions and men. The history of pursuit-evasion games is rich, with the earliest formal problems being inspired by naval exploits [3]. Isaac's text [12] is the classical source for the early survey of the field, with a focus on differential methods. A more recent text by Nahin [25] gives a colloquial overview with more colorful history.

The particular pursuit problem considered in this paper goes under the name of Lion $\mathcal{E}$ Man. The original Lion $\mathcal{E}$ Man problem (attributed to Rado in the 1930s) involves a single pursuer chasing a single evader at equal speeds in continuous time on a domain $\mathcal{D}$ equal to a planar Euclidean disc. In this well-known setting, it was a surprise to find that the evader can win if the pursuer keeps on the radius to the evader (see Littlewood's geometric proof [21] of Besicovich's 1952 result and the subsequent paper of Croft [6]). This problem was generalized by Flynn [8] to account for different speed ratios and solved via differential methods. More recent treatments of the problem appear in $[20,2,33,17]$.

Research supported by DARPA SToMP \# HR0011-07-1-0002 and NSF MSPA-MCS \# 0528086. 
Although pursuit games are traditionally played on a planar Euclidean domain, there are examples of more general playing fields, such as graphs [26], spheres [18, 32], surfaces of revolution [22,10], Euclidean halfspaces [15], hypersurfaces of Euclidean space [31], and general compact CAT(0) metric spaces [1]. The geometry of a playing field has been used as a parameter in proving computational complexity of certain pursuit games [27, 19]. Necessary and sufficient capture criteria are rare, sufficiency being more common in the literature. The focal point of this paper is a necessary and sufficient criterion for capture which applies to general convex Euclidean domains.

1.2. Assumptions. The following are fixed assumptions for all but the final sections of this paper.

(1) $\mathcal{D}$ is a closed unbounded convex Euclidean domain with boundary.

(2) There is a single evader $E$ and $N$ pursuers $\left\{P_{j}\right\}_{1}^{N}$, represented as points with initial locations $E^{0}$ and $\left\{P_{j}^{0}\right\}$ respectively.

(3) For each $t \in \mathbb{N}$, the evader jumps from location $E^{t}$ to $E^{t+1}$, a point within unit distance of $E^{t}$. Then, each pursuer may jump from $P_{j}^{t}$ to $P_{j}^{t+1}$, at most a unit distance.

(4) The evader $E$ wins the game if $P_{j}^{t} \neq E^{t}$ for all $t$ and $j$.

(5) Each pursuer $P_{j}$ has perfect information about $\mathcal{D}$ and about its current position $P_{j}^{t}$ and that of the evader $E^{t+1}$.

The principal result of this paper is a necessary and sufficient condition for the pursuers to win, regardless of evader strategy, as a function of $E^{0},\left\{P_{j}^{0}\right\}$, and $\mathcal{D}$.

1.3. Notation. We fix the following standard notation. Euclidean $n$-dimensional space is denoted $\mathbb{E}^{n}$ with norm $\|\cdot\|$. We choose $n$ so that $\mathcal{D}$ has nonempty interior, that is, $\mathcal{D}$ lies in no hyperplane of $\mathbb{E}^{n}$. The unit sphere in $\mathbb{E}^{n}$ is $S^{n-1}=\left\{x \in \mathbb{E}^{n}:\|x\|=1\right\}$. Given two points $P$ and $Q$ in $\mathbb{E}^{n}$, the line containing them is denoted $P Q$, the segment between them $\overline{P Q}$, and the distance between them $|P Q|$. Assuming $P \neq Q$, let $[P Q] \in$ $S^{n-1}$ denote the unit vector $\overrightarrow{P Q} /|P Q|$. The closed ball of radius $r$ about a point $O \in \mathbb{E}^{n}$ is denoted $B(O, r)$. A cone with central angle $\alpha$ is the union of all rays from a point making angle $\leq \alpha$ with a fixed ray.

We may abuse notation and use $E$ to denote either the evader or an evader's initial position, $E^{0}$. The same holds for pursuers, using $P_{j}$ instead of the more cumbersome $P_{j}^{0}$.

\section{PRIOR AND PRESENT RESUlTS}

We detail known results for Lion $\mathcal{E}$ Man problems, graded as a function of domain characteristics. In all cases, the assumptions of $\$ 1.2$ are in effect. 
2.1. Compact domains. When $\mathcal{D}$ is compact, there is no route of escape, and the evader is always captured. No intricate strategy is required: the greedy strategy of having the pursuer move along the geodesic path to the evader's present location is efficacious if not efficient. For compact convex domains, this is an exercise for the reader. The greedy strategy works as well for any compact CAT(0) domain (a geodesic metric space whose curvature - as measured by comparison triangles - is nowhere positive): see [1] for details.

2.2. No boundaries. In the case where $\mathcal{D}=\mathbb{E}^{n}$, there is an obvious necessary and sufficient criterion for capture:

$(\star) E$ is in the interior of the convex hull of $\left\{P_{j}\right\}_{1}^{N}$.

That this is necessary and sufficient has been discovered and rediscovered in various contexts within the literature. See, in particular, $[6,11,36,17,28]$. The perspective of the present paper is to incorporate the boundary of the domain as a type of stationary pursuer, whose geometry affects the success of a given pursuit strategy. This greatly impacts strategy and feasibility of capture, as it is possible for the pursuers to corner the evader.

2.3. Radius: one pursuer. The paper of Sgall [33] solves a Lion $\mathcal{E}$ Man problem on the closed first quadrant $\mathbf{Q}=\left\{\left(x_{1}, x_{2}\right) \in \mathbb{E}^{2}: x_{i} \geq 0\right\}$. All the assumptions of $\S 1.2$ hold with $\mathcal{D}=\mathbf{Q}$ and $N=1$. For this case, Sgall shows that the pursuer can win if and only if

( $\star$ The set $\{z \in \mathbf{Q}:\|z E\| \leq\|z P\|\}$ is bounded.

The resulting algorithm is denoted Spheres in [17]: we will refer to it as Radius, following the terminology of Croft's 1964 paper [6] (hearkening back to the earlier work of Besicovich, Littlewood, and Rado). The algorithm proceeds as follows. Let $O$ denote a center: a point on the ray with end $E$ through $P$, not between $E$ and $P$, such that the component of $\mathbf{Q}-B(O,|O P|)$ which contains $E$ is bounded. The existence of a center $O$ follows from $(\star)$. The algorithm produces $P^{t+1}$ from $P^{t}$ and $E^{t+1}$ by choosing the point that lies within unit distance of $P^{t}$ and closest to $E^{t+1}$ along the segment $\overline{O E^{t+1}}$.

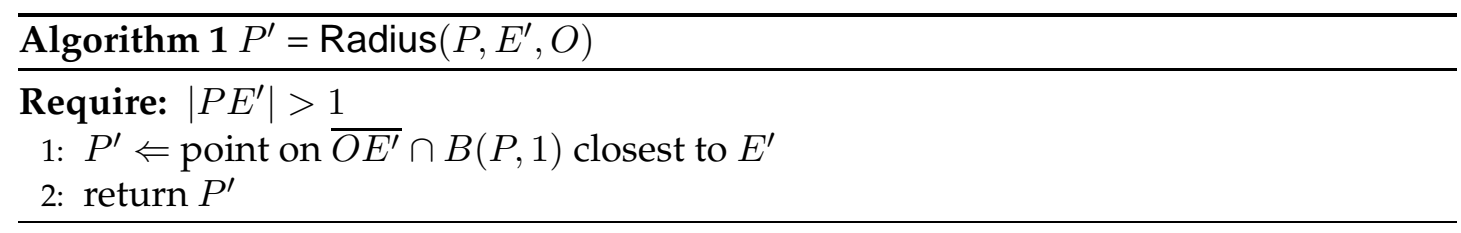

Sgall proves that for $\mathcal{D}=Q$, Radius always succeeds in a finite number of moves for systems satisfying $(\star)$, and that a quadratic number of moves in $|O P|$ is a sharp upper bound. It is remarked at the end of the proof that the result and proof generalize from the quadrant $\mathbf{Q}$ to any planar wedge of angle $<\pi$, as well as to higher-dimensional convex Euclidean cones, with the obvious modification to $(\star)$ above. However, Sgall 
fails to notice that there is a distinction between wedges of angle $<\pi / 2$ and those with angle $\geq \pi / 2$ : for the latter $(\star)$ implies that the center must be in $\mathbf{Q}$, but this is no longer true for the former. We demonstrate in $\S 4.1$ if the angle $<\pi / 2$, his algorithm may fail to work because it may require the pursuer to move outside the domain.

2.4. Radius: multiple pursuers. The paper of Kopparty and Ravishankar [17] considers the broader setting of $N$ pursuers in a convex Euclidean domain in dimension $n$ bounded by finitely many hyperplanes. Their main result is an extension of the proof of Sgall that the Radius algorithm works in this setting. Condition $(\star)$ above generalizes in the following manner:

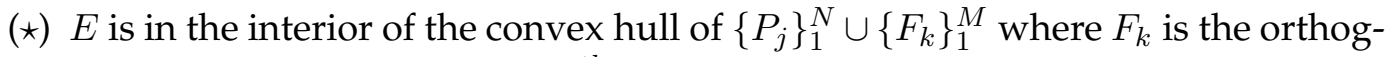
onal projection of $E$ onto the $k^{\text {th }}$ bounding hyperplane of $\mathcal{D}$.

Their algorithm is a simple extension of Sgall's: choose $N$ centers $O_{j}$ for $E$ and the $P_{j}$ such that the set $\mathcal{D}-\cup_{j} B\left(O_{j},\left|O_{j} P_{j}\right|\right)$ has a bounded component containing $E$. Discard the pursuers $P_{j}$ for which the ray from $E$ through $P_{j}$ intersects the boundary of $\mathcal{D}$, and evolve via $P_{j}^{\prime}=\operatorname{Radius}\left(P_{j}, E, O_{j}\right)$. Theorem 3 of [17] claims that Radius leads to successful capture, assuming $(\star)$ holds at time $t=0$ : see Figure 1.

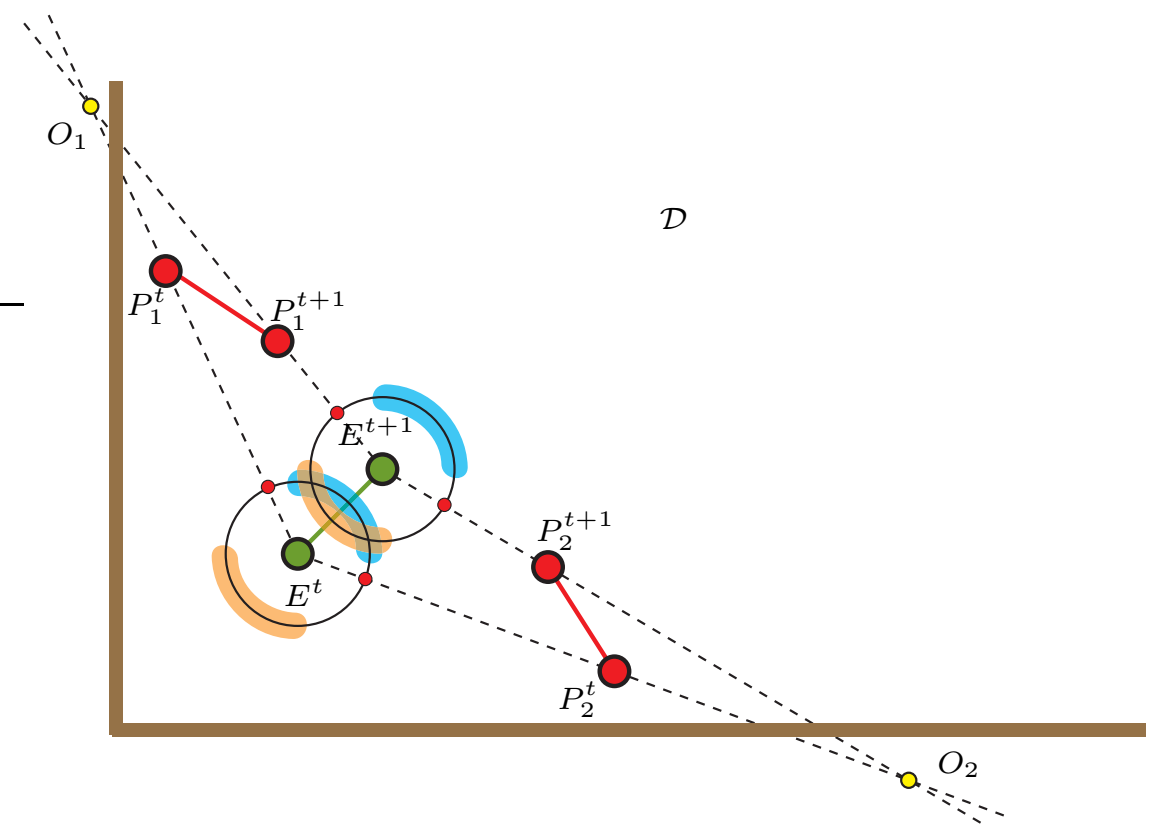

FIGURE 1. One Radius step makes positive progress along the radial direction from center $O_{j}$. (Note: centers $O_{1}, O_{2}$ and lengths not drawn to scale.)

Their paper has additional results, including a modified algorithm called Planes, which is guaranteed to work only in the case of $\mathcal{D}=\mathbb{E}^{n}$ (as does Radius, albeit more slowly). 
2.5. New results. We show the following.

(1) The conditions $(\star$ ) above all generalize to a Boundedness Condition applicable to arbitrary convex Euclidean domains.

(2) The application of Radius is invalid on general convex Euclidean domains (even 2-d cones), contradicting the claims of [33] and [17]. However, there exists a (restrictive) additional geometric assumption under which the proofs of [17] become valid. This condition is not needed when there is a single pursuer and the domain $\mathcal{D}$ diverges to infinity on a wide enough set of directions.

(3) The Boundedness Condition is necessary and sufficient for the existence of a successful pursuit strategy (RotatingRadius) on convex Euclidean domains (not merely those with piecewise-linear boundaries).

(4) The Boundedness Condition gives a sufficient capture criterion on non-convex Euclidean domains which are expressed as a finite union of convex domains. The same algorithm RotatingRadius is played in a parallel projected fashion on the convex components.

All mathematical tools used are very elementary ideas from Euclidean, spherical and convex geometry.

\section{ELEMENTARY GEOMETRY}

This section covers basic definitions from convex geometry [29] and culminates in a general reformulation of the Boundedness Condition. For the remainder of this section, assume $\mathcal{D}$ is a convex Euclidean domain.

Definition 1. A Euclidean domain $\mathcal{D}$ with a configuration of one evader $E$ and $N$ pursuers $\left\{P_{j}\right\}_{1}^{N}$ satisfies the Boundedness Condition if the intersection

$$
\left(\bigcap_{j=1}^{N} \mathbf{H}_{j}\right) \cap \mathcal{D} \text { is bounded, }
$$

where $\mathbf{H}_{j}$ is the closed halfspace containing $E$ whose boundary hyperplane passes through $P_{j}$ orthogonal to $E P_{j}$.

In this section, we reformulate this condition in terms of spherical convexity [7]. (One could just as easily work with cones in the non-normalized setting, but we prefer thinking in terms of visibility spheres.)

Definition 2. A subset $\mathcal{A} \subset S^{n-1}$ of the unit sphere in $\mathbb{E}^{n}$ is said to be convex if the cone over $\mathcal{A}$,

$$
C(\mathcal{A})=\left\{v \in \mathbb{E}^{n}: v=\lambda x \text { for some } x \in \mathcal{A}, \lambda \in[0, \infty)\right\}
$$

is a convex subset of $\mathbb{E}^{n}$.

Remark 3. A great $k$-sphere in $S^{n-1}$, for $0 \leq k \leq n-1$, being by definition the intersection of $S^{n-1}$ with a $(k+1)$-plane through the origin, is a convex subset of $S^{n-1}$ according to our definition. In particular, when $k=0$, a pair of antipodal points is a convex subset. 
Given $\mathcal{D}$, we can encode the constraints imposed by the boundary as well as the possible avenues of escape in terms of dual convex subsets of the unit sphere.

Definition 4. Given $\mathcal{D} \subset \mathbb{E}^{n}$, the normals set, $\mathcal{N}$, is the subset of $S^{n-1}$ containing all the outer unit normal vectors to support hyperplanes in $\mathbb{E}^{n}$ which intersect $\mathcal{D}$ but not its interior.

It is worth noting a point that many authors have misstated: the set of outer unit normals used in this definition is not necessarily convex, though its closure $\mathcal{N}$ is. See [38] for an example where this distinction is critical.

Definition 5. The dual $\mathcal{A}^{0}$ of a convex set $\mathcal{A} \subset S^{n-1}$ is the set of all unit vectors $v$ making angle $\geq \pi / 2$ with every vector in $\mathcal{A}$.

Then $\mathcal{A}^{0}$ is a closed convex set, and $\mathcal{A}^{00}$ is the closure of $\mathcal{A}$.

Definition 6. The recession set of $\mathcal{D}, \mathcal{R}$, is the subset of $S^{n-1}$ containing all unit vectors in the directions of half-lines lying in $\mathcal{D}$.

The following lemma is well known and easy to prove (see [29, p. 123, Corollary 14.2.1]). We denote the closed hemisphere with pole $x$ in $S^{n-1}$ by $\mathcal{H}_{x}$.

Lemma 7. For $\mathcal{D} \subset \mathbb{E}^{n}$ convex, $\mathcal{N}^{0}=\mathcal{R}$. Equivalently, $x \in \mathcal{R}$ if and only if $\mathcal{H}_{-x} \supset \mathcal{N}$.

The encoding of the boundary $(\mathcal{N})$ and the directions of escape $(\mathcal{R})$ are thus dual. In the special case when $\mathcal{N}$ is the empty set (that is, $\mathcal{D}=\mathbb{E}^{n}$ ), its dual $\mathcal{R}=S^{n-1}$ is the entire unit sphere, in accordance with Definition 6. On the other hand, when $\mathcal{D}$ is compact, then $\mathcal{R}$ is empty and $\mathcal{N}=S^{n-1}$.

Lemma 8. For $\mathcal{D} \subset \mathbb{E}^{n}$ convex, $\mathcal{N}$ lies in a closed hemisphere of $S^{n-1}$ if and only if $\mathcal{D}$ is unbounded.

Proof. The closure of $\mathcal{N}$, being convex and nonempty, either lies within a closed hemisphere of $S^{n-1}$ or coincides with $S^{n-1}$. By Lemma 7, the latter case occurs if and only if $\mathcal{R}$ is empty, hence if and only if $\mathcal{D}$ is bounded.

The following is a general reformulation of the Boundedness Condition in terms of recession sets and normals sets. Recall $\left[E P_{j}\right]$ denotes the unit vector in the direction from $E$ to $P_{j}$.

Theorem 9. The following are equivalent:

(1) $\mathcal{D} \subset \mathbb{E}^{n}$ satisfies the Boundedness Condition.

(2) No closed hemisphere of $S^{n-1}$ contains $\mathcal{N} \cup\left\{\left[E P_{j}\right]\right\}_{1}^{N}$.

(3) The union of the open hemispheres in $S^{n-1}$ with poles $\left[E P_{j}\right]$ contains $\mathcal{R}$.

(4) The spherical convex hull of $\mathcal{N} \cup\left\{\left[E P_{j}\right]\right\}_{1}^{N}$ equals $S^{n-1}$. 


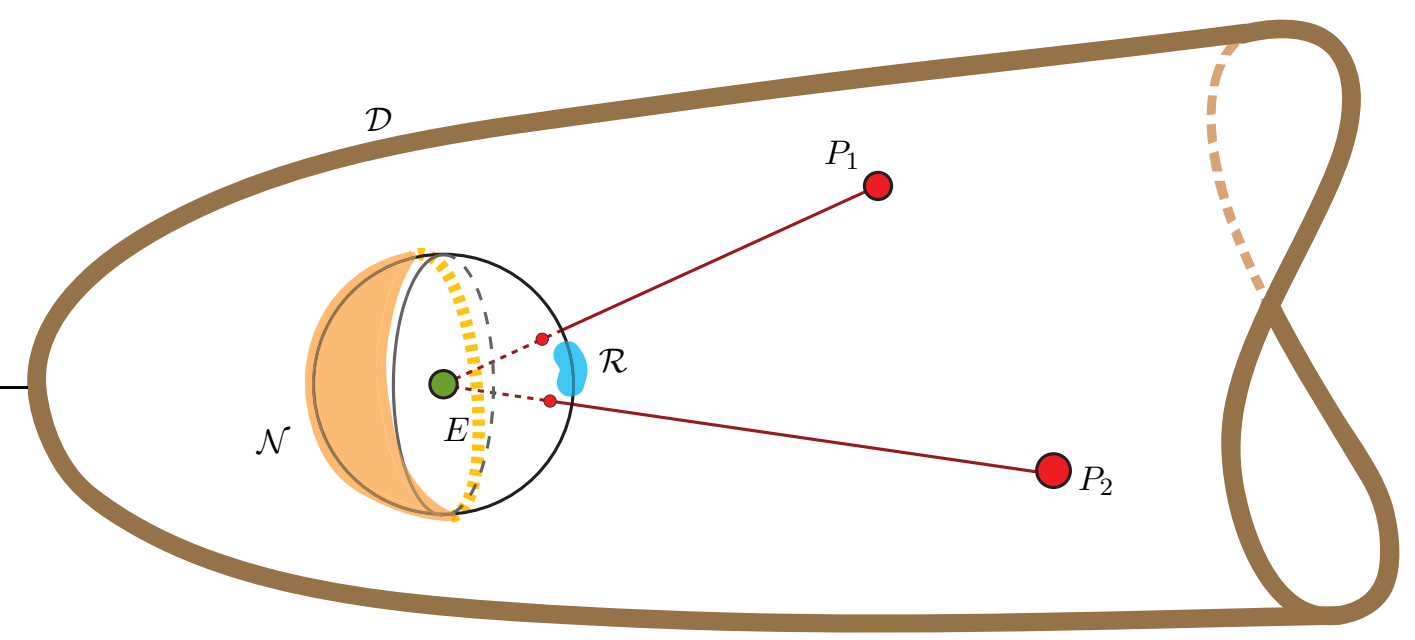

FIGURE 2. The geometry of the domain and the pursuers is encoded in the visibility (unit tangent) sphere at $E$ : pictured is the recession set $\mathcal{R}$, its dual the normals set $\mathcal{N}$, and the perceived locations of the pursuers $\left[E P_{j}\right]$. The Boundedness Condition is equivalent to saying that the spherical convex hull of $\mathcal{N}$ and the $\left[E P_{j}\right]$ is the entire visibility sphere.

Proof. The equivalence of the Boundedness Condition with (2) above follows from Lemma 8 applied to the set given by the intersection of $\mathcal{D}$ with the appropriate halfspaces as in Definition 1. The equivalence of Conditions (2) and (3) follows from Lemma 7 , since $x \in \cup_{j}$ int $\mathcal{H}_{\left[E P_{j}\right]}$ for every $x \in \mathcal{R}$ if and only if for every hemisphere $\mathcal{H}_{-x}$ containing $\mathcal{N}$ we have $\left[E P_{j}\right] \in \operatorname{int} \mathcal{H}_{x}$ for some $j$. The equivalence of (2) and (4) follows from Definition 2.

Note that this result specializes in the case of $\mathcal{D}=\mathbb{E}^{n}$ : the evader must lie in the interior of the convex hull of the pursuers.

The Boundedness Condition means that the evader cannot simultaneously move away from the boundary of the playing field and all the pursuers. If the evader ever can, then, of course, the evader wins.

Proposition 10. The Boundedness Condition is a necessary condition for the existence of a successful pursuit strategy.

Proof. If the Boundedness Condition fails, then all of the vectors $\left[E P_{j}\right]$ together with $\mathcal{N}$ lie within a single hemisphere $\mathcal{H}$ of $S^{n-1}$, thanks to Theorem 9. Let $v$ be the unique vector in $S^{n-1}$ dual to $\mathcal{H}$. By definition, $v \in \mathcal{R}$. Moving $E$ in the direction $v$ has an infinite trajectory which furthermore never decreases the distance to any $P_{j}$ (as a trivial calculation shows). 


\section{BOUNDARY EFFECTS AND Radius}

We consider carefully under which circumstances the Radius algorithm of $[17,33]$ is valid and effective.

4.1. When Radius fails. The first step of Radius in [17, p. 120] is to discard all the pursuers $P_{j}$ for which the ray $E P_{j}$ intersects a bounding hyperplane of $\mathcal{D}$, or equivalently, for which $\left[E P_{j}\right] \notin \mathcal{R}$. However, the Boundedness Condition may fail to be preserved under this step; worse, all the pursuers may be discarded. On the other hand, if the discarding step is omitted, the algorithm may move pursuers out of the playing field. Figure 3 gives a planar example involving a single pursuer.
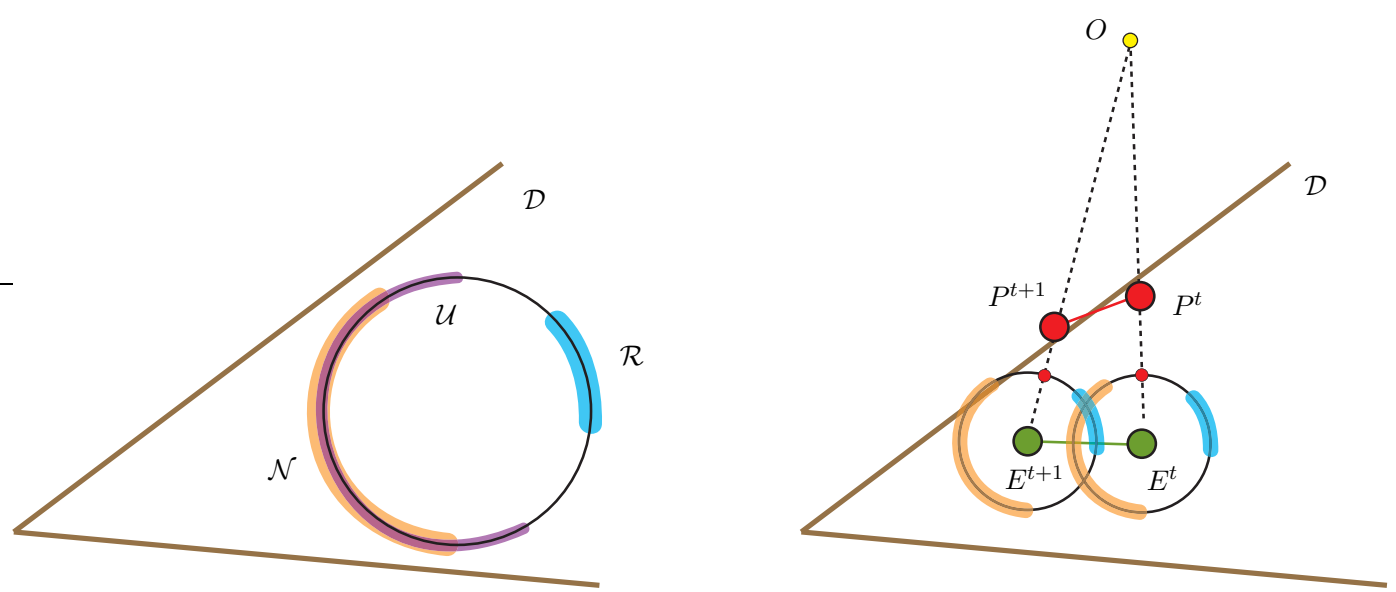

FIGURE 3. For a thin cone, the recession set $\mathcal{R}$ and the hemispheres set $\mathcal{U}$ (see Definition 14) do not partition the unit tangent sphere [left]. Consequently, the Radius algorithm can fail, in this case [right] by demanding that $P^{t+1}$ lie outside of $\mathcal{D}$. (Note: the point $O$ is not drawn at the appropriate distance to $\mathcal{D}$ for scale purposes.)

Remark 11. It may be argued that the chances of having pursuers 'tricked' into a boundary collision via Radius is rare; or that this is a result of a degenerate set of initial conditions; or still that the difference between where Radius and where the laws of physics demand a pursuer go are too small to affect the outcome of the game. In dimension two, such consideration might have validity. However, as the dimension of the domain $\mathcal{D}$ increases, the possibilities for mischief on the part of an adversarial evader increase dramatically. Consider the example of a domain $\mathcal{D}$ whose recession set is very thin. For example, in 3-d, this would correspond to a domain with minimal cone angle near zero and maximal cone angle near $\pi$, as in Figure 4. In the case of several pursuers beginning near the boundary and which just barely satisfy the Boundedness Condition, it is possible for the evader to 'zig-zag' and force pursuers to collide into the boundary at many/all time steps. Small errors in progress induced by these boundary effects could presumably accumulate under such an evader strategy. 


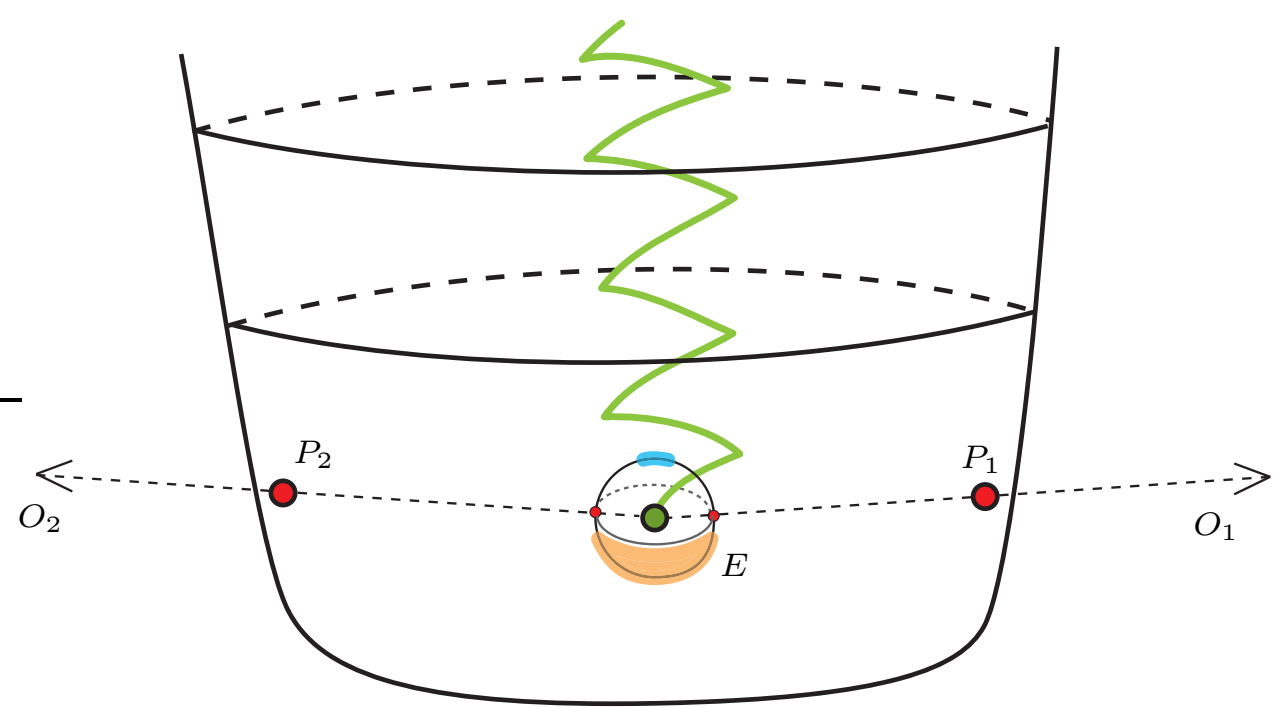

FIGURE 4. A higher-dimensional convex domain with a thin but long recession set could lead to a situation in which boundary collisions are prevalent.

Further generalizations of this example to domains which have several independent cone angles close to $\pi$ along with several close to zero could prove more challenging, since the evader has multiple directions in which to escape, while pursuers can experience a boundary collision at many time steps.

4.2. When Radius works. If no pursuers are discarded but the evader always moves so that the Radius algorithm leaves them in $\mathcal{D}$, then the evader is captured. Thus in Figure 1, even if the evader moves steadily in the recession direction that makes angle $\pi / 4$ with the positive $x$ and $y$ axes, the escape route will be blocked. The following is a corrected version of Theorem 3 of [17]. The proof that Radius works in this restricted case follows the proof of [17] exactly. We include a careful proof for the sake of completeness.

Theorem 12. For any convex unbounded $\mathcal{D}$, the pursuers win if (1) the Boundedness Condition holds and (2) $\left[E P_{j}\right] \in \mathcal{R}$ for all $j$.

Proof. Condition (2) implies that $O_{j} \in \mathcal{D}$ for all $j$, and $P_{j}^{t+1}=\operatorname{Radius}\left(P_{j}^{t}, E_{j}^{t+1}, O_{j}\right)$ returns a value in $\mathcal{D}$, since $P_{j}^{t+1}$ lies on the segment $\overline{O_{j} E^{t+1}} \subset \mathcal{D}$. Since the colinearity and order of the triples $\left(E^{t}, P_{j}^{t}, O_{j}\right)$ are maintained as a function of $t$, the evader must remain for all $t$ within the (bounded!) domain of Eqn. (1). However, since the angle $\angle O P_{j}^{t} P_{j}^{t+1}$ is obtuse and $\left|P_{j}^{t} P_{j}^{t+1}\right|=1$, the Law of Cosines implies that

$$
\left|O_{j} P_{j}^{t+1}\right|^{2}>\left|O_{j} P_{j}^{t}\right|^{2}+1
$$


implying the eventual capture of $E$.

Remark 13. It is permissible to discard any number of pursuers $a b$ initio, so long as the Boundedness Condition holds with the remaining pursuers.

In the case of a single pursuer $P$, we can present a simple condition on the playing field $\mathcal{D}$ that guarantees the success of Radius.

Definition 14. For a fixed domain $\mathcal{D}$, let $\mathcal{U}$ denote the union of all closed hemispheres in $S^{n-1}$ that contain $\mathcal{N}$.

By Theorem 9, the Boundedness Condition in the single pursuer case becomes $[E P] \notin$ $\mathcal{U}$.

Lemma 15. Assume there is a single pursuer $P$, and that $\mathcal{R} \cup \mathcal{U}=S^{n-1}$. Then the pursuer wins following Radius if the Boundedness Condition holds.

Proof. Since $\mathcal{R} \cup \mathcal{U}=S^{n-1}$, the Boundedness Condition $[E P] \notin \mathcal{U}$ implies $[E P] \in \mathcal{R}$, and so the hypotheses of Theorem 12 are satisfied.

If the condition of Lemma 15 fails, then Radius may fail, as the planar example in Figure 3 illustrates.

Theorem 16. In the case of a single pursuer, if $\mathcal{D}$ contains a cone with central angle at least $\pi / 4$, then the Boundedness Condition guarantees capture via Radius.

Proof. Suppose $\mathcal{D}$ contains a cone with central angle $\pi / 4$. Equivalently, $\mathcal{R}$ contains a spherical disk of radius $\pi / 4$ centered on some unit vector $v$ (that is, all unit vectors making angle $\leq \pi / 4$ with $v$ ). Since $\mathcal{R}$ and $\mathcal{N}$ are dual, the set $\mathcal{N}$ must be contained in a spherical disk of radius $\pi / 4$ about $-v$. It follows that $\mathcal{U}$, the union of all closed hemispheres containing $\mathcal{N}$, contains the spherical disk of radius $3 \pi / 4$ about $-v$. Therefore $\mathcal{R} \cup \mathcal{U}=S^{n-1}$, as desired. Lemma 15 completes the proof.

When $\mathcal{D}$ does not contain a sufficiently large subcone, it is still possible to ensure capture, as we demonstrate in the next section.

\section{SufFICIENCY OF THE BOUNDEDNESS CONDITION}

We introduce Algorithm RotatingRadius to resolve the deficiencies of Radius and provide a complete characterization of when capture is possible. From Theorem 12 we see that the Radius algorithm works if $\left\{\left[E P_{j}\right]\right\}_{1}^{N} \subset \mathcal{R}$. In this case, under the Radius algorithm, each pursuer $P_{j}$ computes a center $O_{j}$ on the line $E P_{j}$ and moves radially away from this center. The centers $O_{j}$ are fixed throughout the game, and the evader is blocked from entering a family of expanding concentric spheres about each $O_{j}$. 
However, in the case where $\left[E P_{j}\right] \notin \mathcal{R}$, Radius may move a pursuer $P_{j}^{t}$ to a position $P_{j}^{t+1}$ outside $\mathcal{D}$. When this occurs, the strategy of the RotatingRadius algorithm is to recalculate $P_{j}^{t+1}$ to lie in $\mathcal{D}$, using nearest-point projection. RotatingRadius then recalculates the center $O_{j}^{t+1}$, changing the blocking sphere so that the new one continues to contain the old one even though they are no longer concentric. The key is to show this may be done while keeping the radii of the blocking spheres bounded.

Theorem 17. Discrete-time equal-speed capture on a convex domain $\mathcal{D}$ is achievable if and only if the initial positions of the pursuers and evader satisfy the Boundedness Condition.

5.1. The RotatingRadius algorithm. One begins by discarding those pursuers $\left\{P_{j}\right\}$ for which $\left[E P_{j}\right] \in \mathcal{N}$. By Theorem 9, this move preserves the Boundedness Condition. After the evader moves from $E^{t}$ to $E^{t+1}$, each pursuer $P_{j}^{t}$ and its corresponding center $O_{j}^{t}$ is updated according to $\left(P_{j}^{t+1}, O_{j}^{t+1}\right)=$ RotatingRadius $\left(P_{j}^{t}, E^{t}, O_{j}^{t}, \mathcal{D}\right)$. See Figure 5.

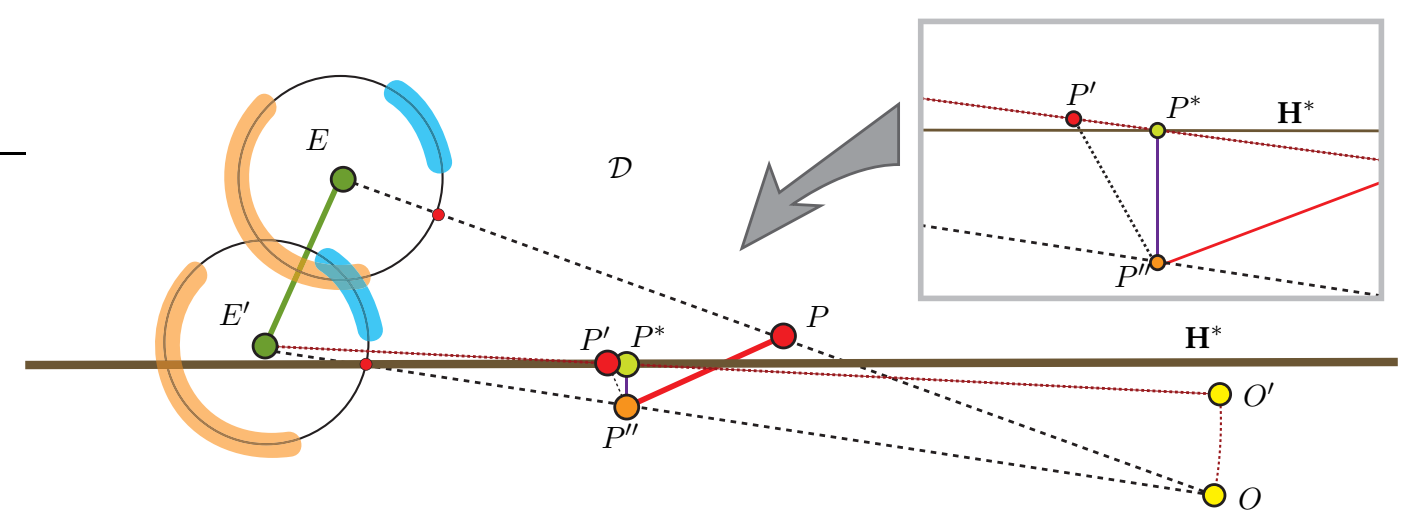

FIGURE 5. The RotatingRadius algorithm deals with boundary collisions in Radius by projecting $P^{\prime \prime}$ to $P^{*}$ in the boundary $\partial \mathcal{D}$ and then moving the center $O$ to $O^{\prime}$ with $P^{\prime}$ placed along $\overline{E^{\prime} O^{\prime}}$.

5.2. Radial progress. For Radius, Sgall bases his estimate of capture time on the following estimate, which we adapt to RotatingRadius.

Lemma 18. If $P_{j}^{t+1} \neq E^{t+1}$,

$$
\left|O_{j}^{t} P_{j}^{t}\right|^{2}+1<\left|O_{j}^{t+1} P_{j}^{t+1}\right|^{2}
$$

Proof. Set $P=P_{j}^{t}$. Since $\left|E E^{\prime}\right| \leq 1$, the distance from any point $M$ of the interior of the segment $\overline{E^{\prime} O}$ to the line $E O$ is $<1$. Taking $M$ such that $P M \perp E O$ we see that there are two points on $E^{\prime} O$ at distance 1 from $P$, and the one, $P^{\prime \prime}$, nearest to $E^{\prime}$ forms an obtuse angle $\angle O P P^{\prime \prime}$. Hence by the Law of Cosines, $|O P|^{2}+1<\left|O P^{\prime \prime}\right|^{2}$. If $P^{\prime \prime} \in \mathcal{D}$, then $P_{j}^{t+1}=P^{\prime \prime}$ and we are done. 


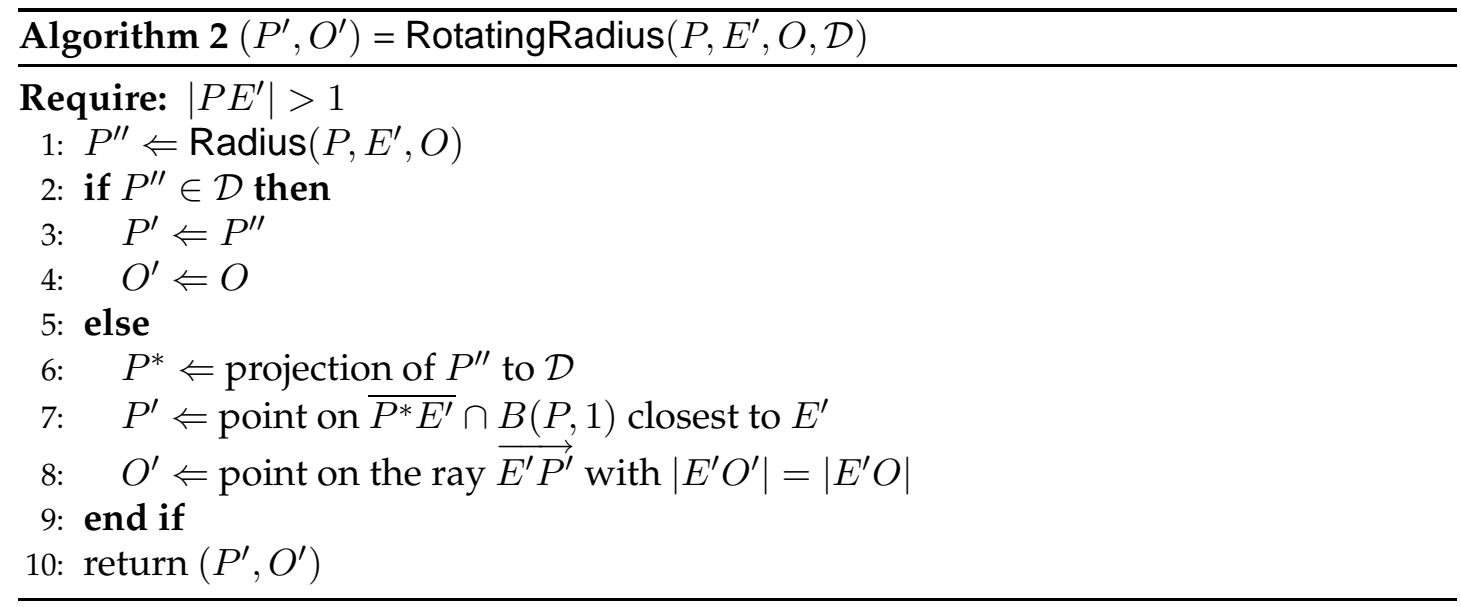

Otherwise we continue the algorithm by letting $P^{*}$ be the nearest point in $\mathcal{D}$ to $P^{\prime \prime}$ (clearly $\left|P P^{*}\right|<1$ ), and letting $\mathbf{H}^{*}$ denote the halfspace containing $\mathcal{D}$ and bounded by the support hyperplane to $\mathcal{D}$ at $P^{*}$ that is orthogonal to $P^{\prime \prime} P^{*}$. Then $P^{\prime}=P_{j}^{t+1}$ is the unique point on the segment $\overline{E^{\prime} P^{*}}$ at distance 1 from $P$. Since $E^{\prime} \in \mathcal{D}$ and $P^{\prime \prime} \notin \mathcal{D}$, then $E^{\prime}$ is in $\mathbf{H}^{*}$ and so $\angle E^{\prime} P^{*} P^{\prime \prime} \geq \pi / 2$. Hence $\left|P^{*} E^{\prime}\right|<\left|P^{\prime \prime} E^{\prime}\right|$. Since $\left|E^{\prime} O^{\prime}\right|=\left|E^{\prime} O\right|$, we have

$$
\left|O^{\prime} P^{\prime}\right|^{2}>\left|O^{\prime} P^{*}\right|^{2}>\left|O P^{\prime \prime}\right|^{2}>|O P|^{2}+1
$$

5.3. A decreasing playing field. Consider the closed ball $B_{j}^{t}=B\left(O_{j}^{t},\left|O_{j}^{t} P_{j}^{t}\right|\right)$. Let $\mathcal{C}^{t}$ be the component of $\left(\mathcal{D}-\cup_{j} B_{j}^{t}\right)$ containing $E^{t}$. We prove that $\mathcal{C}^{t}$ is strictly monotonically decreasing under the RotatingRadius algorithm, thus providing a set-valued Lyapunov function.

Lemma 19. $\mathrm{cl}^{t+1} \subset \mathcal{C}^{t}$ for every $t$.

Proof. In this proof, we fix $j$ and continue the notation of $\S 5.1$. Set $B^{t}=B_{j}^{t}=B\left(O_{j}^{t},\left|O_{j}^{t} P_{j}^{t}\right|\right)$, and let $S^{t}$ be the boundary sphere of $B^{t}$. When $P^{\prime \prime}=P_{j}^{t+1}$, we have $O=O^{\prime}$, so by Lemma $18, B^{t+1}$ is concentric with and larger than $B^{t}$. Otherwise we show that the ball $B^{t+1}$ about $O^{\prime}$ includes the intersection of $\mathcal{D}$ with the ball $B^{t}$ about $O$, that is,

$$
\left(B^{t+1} \cap \mathcal{D}\right) \supset\left(B^{t} \cap \mathcal{D}\right) \text {. }
$$

Consider two concentric spheres with center $O^{\prime}: S^{t+1}$ through $P^{\prime}$ and $S$ with radius $\left|O P^{\prime \prime}\right|$. We also have two concentric spheres with center $O: S^{t}$ through $P$ and $S^{\prime \prime}$ through $P^{\prime \prime}$. By (2), the corresponding balls satisfy $B^{t+1} \supset B$ and $B^{\prime \prime} \supset B^{t}$. Therefore (3) will follow from

$$
(B \cap \mathcal{D}) \supset\left(B^{\prime \prime} \cap \mathcal{D}\right) .
$$


Since $S$ and $S^{\prime \prime}$ have the same radius, their intersection $S \cap S^{\prime \prime}$ is an $(n-2)$-sphere $S^{\prime}$ centered at the midpoint of the segment $\overline{O O^{\prime}}$ and lying in the perpendicular bisecting hyperplane of that segment. Let $\mathbf{H}^{\prime}$ be the halfspace containing $O^{\prime}$ and bounded by this perpendicular bisector. Then

$$
\left(B \cap \mathbf{H}^{\prime}\right) \supset\left(B^{\prime \prime} \cap \mathbf{H}^{\prime}\right) .
$$

Therefore (4) will follow in turn from

$$
\left(B^{\prime \prime} \cap \mathcal{D}\right) \subset \mathbf{H}^{\prime}
$$

since then

$$
(B \cap \mathcal{D}) \supset\left(B \cap \mathbf{H}^{\prime} \cap \mathcal{D}\right) \supset\left(B^{\prime \prime} \cap \mathbf{H}^{\prime} \cap \mathcal{D}\right) \supset\left(B^{\prime \prime} \cap \mathcal{D}\right) .
$$

By construction, $\overline{P^{\prime \prime} P^{*}}$ is the shortest join from $P^{*}$ to $\mathcal{D}$, so $\mathcal{D}$ lies in the half-space $\mathbf{H}^{*}$ bounded by the support hyperplane to $\mathcal{D}$ at $P^{*}$ that is orthogonal to $P^{\prime \prime} P^{*}$. Thus (5) will follow from

$$
\left(B^{\prime \prime} \cap \mathbf{H}^{*}\right) \subset \mathbf{H}^{\prime},
$$

which we now verify.

The centers $O$ and $O^{\prime}$ of $B^{\prime \prime}$ and $B$, respectively, and the point $E^{\prime}$ all lie on a 2-plane $T$ orthogonal to the bounding hyperplane of $\mathbf{H}^{\prime}$. Moreover, $T$ is also orthogonal to the bounding hyperplane of $\mathbf{H}^{*}$ since $P^{\prime \prime}$ and $P^{*}$ lie on $T$. By symmetry, it suffices to verify (6) when $B^{\prime \prime}, \mathbf{H}^{*}$ and $\mathbf{H}^{\prime}$ are reinterpreted as their respective intersections with $T$.

Thus we regard $\mathbf{H}^{*}$ as a halfplane bounded by a support line at $P^{*}$ for $\mathcal{D} \cap T$, and $\mathbf{H}^{\prime}$ as the halfplane containing $O^{\prime}$ bounded by the perpendicular bisecting line of $\overline{O O^{\prime}}$. Similarly, $S \cap S^{\prime \prime}$ is an intersection of two circles, of equal radius and with centers at equal distance from $E^{\prime}$, and consists of two points on the bisecting line.

Because $\angle E^{\prime} P^{*} P^{\prime \prime}$ is obtuse, $\left|E^{\prime} P^{*}\right|<\left|E^{\prime} P^{\prime \prime}\right|$. Since $\left|E^{\prime} O\right|=\left|E^{\prime} O^{\prime}\right|$, then $\left|O^{\prime} P^{*}\right|>$ $\left|O P^{\prime \prime}\right|$, that is, $P^{*}$ lies outside $B$. Since $P^{*} \in \mathbf{H}^{\prime}$ and $(B \cap H) \supset\left(B^{\prime \prime} \cap H\right)$, then $P^{*}$ lies outside $B \cup B^{\prime \prime}$. Therefore the segment segment $\overline{P^{*} P^{\prime \prime}}$ lies except for its righthand endpoint outside $B \cup B^{\prime \prime}$. It follows that $B^{\prime \prime} \cap \mathbf{H}^{*}$ cannot leave $\mathbf{H}^{\prime}$, since on the bounding line of $\mathbf{H}^{\prime}$, the intersection point with the bounding line of $\mathbf{H}^{*}$ is separated from $S \cap S^{\prime \prime}$ by the intersection point with $\overline{P^{*} P^{\prime \prime}}$.

\subsection{Proof of Theorem 17.}

Proof. If $O_{j}^{t+1} \neq O_{j}^{t}$, the closed halfspace $\mathbf{H}_{j}^{t+1}$ containing $O_{j}^{t+1}$ and bounded by the perpendicular bisecting hyperplane of $\overline{O_{j}^{t} O_{j}^{t+1}}$ consists of the points of $\mathbb{E}^{n}$ that are no further from $O_{j}^{t+1}$ than from $O_{j}^{t}$.

Choose a point $Q_{j} \in\left(B_{j}^{0} \cap \mathcal{D}\right)$. By (3), $Q_{j} \in\left(B_{j}^{t} \cap \mathcal{D}\right)$ for all $t$. Thus, in the notation of the preceding section, we always have $Q_{j} \in B^{\prime \prime}{ }_{j}$. Since $Q_{j} \in \mathcal{D}$ and $\mathbf{H}_{j}^{*}$ is a supporting 


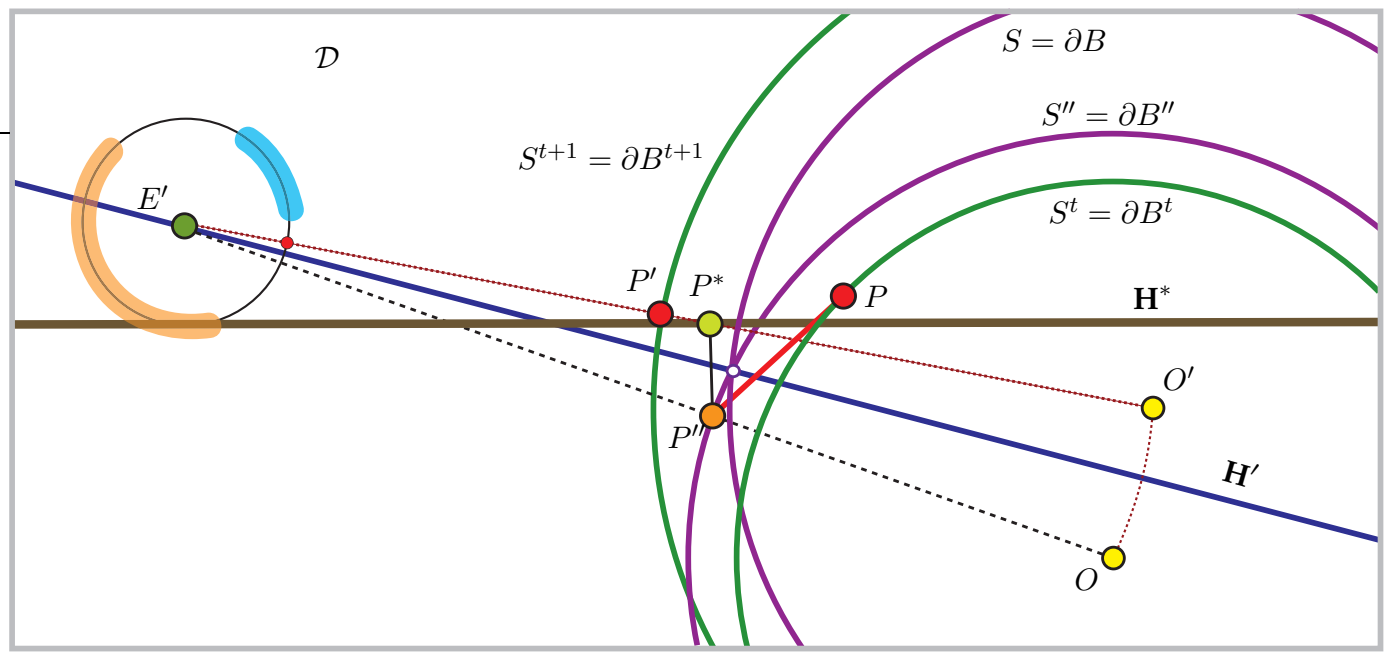

FIGURE 6. Progress in the RotatingRadius algorithm is proved by demonstrating a nestedness property for balls $B^{t}$ intersected with $\mathcal{D}$.

halfspace for $\mathcal{D}$, we also have $Q_{j} \in \mathbf{H}_{j}^{*}$. But then by (6), $Q_{j} \in \mathbf{H}_{j}^{t+1}$ for all $t$. Therefore the distance $\left|Q_{j} O_{j}^{t}\right|$ is nonincreasing in $t$.

The lengths $\left|Q_{j} O_{j}^{t}\right|$ are uniformly bounded for all $t$, as are the lengths $\left|Q_{j} E^{t}\right|$ by Lemma 19. Therefore the lengths $\left|O_{j}^{t} E^{t}\right|$ are uniformly bounded in $t$ as well. By Lemma 18,

$$
\left|O_{j}^{t} E^{t}\right|^{2} \geq\left|O_{j}^{t} P_{j}^{t}\right|^{2} \geq\left|O_{j}^{0} P_{j}^{0}\right|^{2}+t .
$$

Therefore capture occurs.

5.5. Quadratic estimate. The proof of Theorem 17 yields the following estimate, just as in the setting of $[33,17]$.

Corollary 20. Under the Boundedness Condition, if $Q_{j} \in\left(B_{j}^{0} \cap \mathcal{D}\right)$, then the pursuers catch the evader in time

$$
t<\min _{j}\left[\left(\left|Q_{j} O_{j}^{0}\right|+\max \left\{\left|Q_{j} x\right|: x \in \mathcal{C}_{j}^{0}\right\}\right)^{2}-\left|O_{j}^{0} P_{j}^{0}\right|^{2}\right] .
$$

Proof. For each $j$, the time of capture $t$ satisfies

$$
\left|O_{j}^{0} P_{j}^{0}\right|^{2}+t<\left|O_{j}^{t} P_{j}^{t}\right|^{2} \leq\left(\left|O_{j}^{t} Q_{j}\right|+\left|Q_{j} P_{j}^{t}\right|\right)^{2} \leq\left(\left|Q_{j} O_{j}^{0}\right|+\max \left\{\left|Q_{j} x\right|: x \in \mathcal{C}_{j}^{0}\right\}\right)^{2} .
$$

The first inequality is from (7), and the last is by the nonincreasing property of $\left|Q_{j} O_{j}^{t}\right|$ and Lemma 19. 


\section{NONCONVEX DOMAINS: CONVEX DECOMPOSITION}

The tools used in the proofs of this paper are intimately linked to convexity, making the prospects for extending Theorem 17 to arbitrary Euclidean domains seem dim. However, by fixing a convex decomposition of a more general domain and using properties of projections to convex sets, it is possible to give a surprisingly broad generalization.

6.1. The Extended Boundedness Condition. Consider a domain $\mathcal{D}$ in $\mathbb{E}^{n}$, again with a configuration of one evader $E$ and $N$ pursuers $\left\{P_{j}\right\}$. Suppose $\mathcal{D}$ is expressible as a union $\mathcal{D}=\cup \mathcal{D}_{\alpha}$ where each $\mathcal{D}_{\alpha}$ is a convex domain with boundary. We assume neither that this union is disjoint, nor that each $\mathcal{D}_{\alpha}$ is noncompact, nor that each $\mathcal{D}_{\alpha}$ is $n$-dimensional.

The assumptions on the motion of the pursuers and evader must be modified slightly in the non-convex setting. In particular, the unit-distance upper bound on the distance moved per time step must now be interpreted within the interior geometry of $\mathcal{D}$. Players may move to the endpoint of any (rectifiable) path in $\mathcal{D}$ of at most unit length from the start point: players may not "jump" across corners or other boundary features.

Definition 21. Let $\mathrm{PROJ}_{\alpha}: \mathbb{E}^{n} \rightarrow \mathcal{D}_{\alpha}$ denote nearest-point projection to $\mathcal{D}_{\alpha}$. The maps $\mathrm{PROJ}_{\alpha}$ are well-defined projections, since $\mathcal{D}_{\alpha}$ is convex. The Extended Boundedness Condition states that the set of pursuers can be partitioned into nonempty collections $\left\{P_{\alpha j_{\alpha}} \in \mathcal{D}_{\alpha}, 1 \leq\right.$ $\left.j_{\alpha} \leq N_{\alpha}\right\}$, where for each noncompact $\mathcal{D}_{\alpha}$, the configuration of the evader $\operatorname{PROJ}_{\alpha}(E)$ and $N_{\alpha}$ pursuers $\left\{P_{\alpha j_{\alpha}}\right\}$ satisfies the Boundedness Condition.

Note that for compact $\mathcal{D}_{\alpha}$, the Extended Boundedness Condition merely says $\mathcal{D}_{\alpha}$ contains at least one designated pursuer.

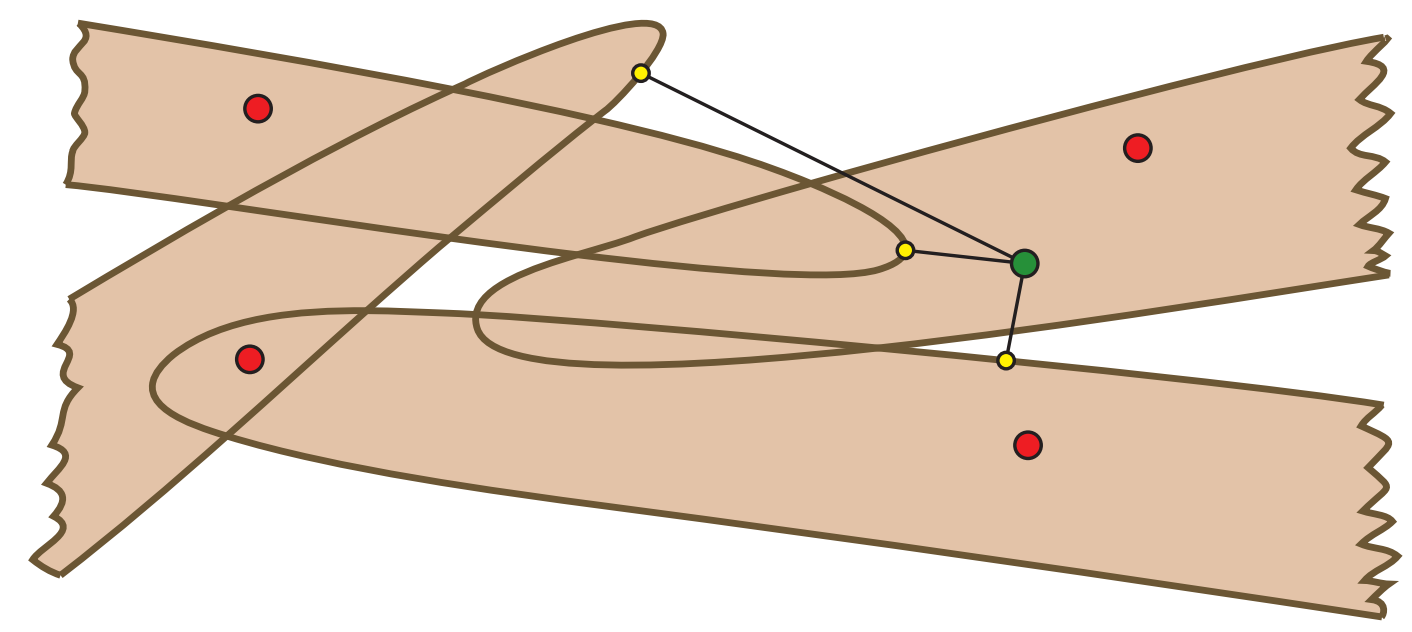

FIGURE 7. On a domain with convex decomposition, one projects the evader's position onto convex factors and plays pursuit games in parallel. 
Theorem 22. The Extended Boundedness Condition is sufficient to ensure discrete-time equalspeed capture on $\mathcal{D}$.

Proof. Since the maps $\mathrm{PROJ}_{\alpha}$ are projections, they are distance nonincreasing, and consequently the jumps of $\operatorname{PROJ}_{\alpha}(E)$ are at most unit distance. For each noncompact $\mathcal{D}_{\alpha}$, let the $N_{\alpha}$ pursuers $\left\{P_{\alpha j_{\alpha}}\right\}$ follow the RotatingRadius algorithm applied to $\mathcal{D}_{\alpha}$ with evader $\operatorname{PROJ}_{\alpha}(E)$. If $\mathcal{D}_{\alpha}$ is compact, set $O_{\alpha j_{\alpha}}^{0}=P_{\alpha j_{\alpha}}^{0} \in \mathcal{D}$ and continue as in Radius. If a pursuer $P_{\alpha j_{\alpha}}$ captures $\operatorname{PROJ}_{\alpha}(E)$ but $\operatorname{PROJ}_{\alpha}(E) \neq E$, thereafter let $P_{\alpha j_{\alpha}}$ move where $\operatorname{PROJ}_{\alpha}(E)$ moves, namely $P_{\alpha j_{\alpha}}^{t}=\operatorname{PROJ}_{\alpha}\left(E^{t}\right)$ for subsequent $t$.

In accordance with the estimates in the preceding section, the projected or 'ghost' evader $\operatorname{PROJ}_{\alpha}(E)$ is eventually captured for each $\alpha$. However, $\operatorname{PROJ}_{\alpha}(E)=E$ for at least one $\alpha$ : $E$ is captured.

6.2. Fewer pursuers. The Extended Boundedness Condition is dependent upon a choice of convex decomposition. An infelicitous choice (too many components) leads to an excessive lower bound on the number of pursuers needed.

In addition, the analogue of Proposition 10 does not hold in this context. If the Extended Boundedness Condition fails for a given decomposition (or even for any convex decomposition), it does not imply that capture cannot be achieved.

Remark 23. As in $\S 5.3$, for each $\mathcal{D}_{\alpha}$, let $\mathcal{C}_{\alpha}^{t}$ be the component of $\left(\mathcal{D}_{\alpha}-\cup_{j_{\alpha}} B_{j_{\alpha}}^{t}\right)$ containing $\operatorname{PROJ}_{\alpha}\left(E^{t}\right)$. Say $\mathcal{C}_{\alpha}^{t}$ and $\mathcal{C}_{\beta}^{t}$ are accessible from each other if and only if the interior distance in $\mathcal{D}$ between them is $\leq 1$. The possible locations for $E^{t+1}$ are in those domains $\mathcal{C}_{\beta}^{t}$ that are accessible from some $\mathcal{C}_{\alpha}^{t}$ for which $E^{t} \in \mathcal{D}_{\alpha}$. Consider the graph $\Gamma^{t}$ whose vertices $V_{\alpha}$ correspond to the domains $\mathcal{D}_{\alpha}$, and whose edges correspond to the accessible pairs $\left\{\mathcal{C}_{\alpha}^{t}, \mathcal{C}_{\beta}^{t}\right\}$. By Lemma 19, at each step no new edges are created while some may be lost. Thus at step $t$, we can discard all the designated pursuers for all the domains $\mathcal{D}_{\beta}$ such that $V_{\beta}$ does not lie in the same connected component of $\Gamma^{t}$ as any $V_{\alpha}$ satisfying $E^{t} \in \mathcal{D}_{\alpha}$.

\section{CONCLUDING REMARKS}

We close with a sequence of remarks delineating extensions, open problems, and significant aspects of the techniques here introduced.

Remark 24. General domains. We stress that the difficulties handled in this paper all stem from the combination of dimensionality and constraints in the domains considered. In general, 2-dimensional playing fields are fairly easy to deal with (the proof of Theorem 17 can be greatly simplified in the planar case). High-dimensional playing fields without boundary are trivial. It is the combination of a potentially intricate, high-dimensional boundary which provides the core challenge. There is seemingly no 
hope of adapting differential game-theoretic methods to such problems (since changing the boundary induces subtle global constraints), and we are left with geometry as a recourse. Fortunately, there are sufficient geometric tools available.

Remark 25. Recession sets. An important contribution of this paper is the recognition of the recession set as a means of encoding domain geometry in pursuit problems. This allows one to speak of the evader-pursuer sightlines, the boundary normals of the domain, and the available escape routes in a common context - subsets of the unit tangent sphere. The combination of Theorems 9 and 17 imply that feasibility of capture is a function of the geometry of the recession set relative to evader-pursuer sightlines. Moreover, the pursuers' plan may be viewed as an attempt to move the vectors $\left[E P_{j}\right]$ into the recession set while preserving the boundedness condition.

Remark 26. Multiple evaders. We have considered multi-pursuer games with a single evader. Consider a modification to the assumptions of $\S 1.2$ in which there are $M$ evaders $E_{\ell}$ moving in discrete time along the sequences $\left\{E_{\ell}^{t}\right\}$ with $\left|E_{\ell}^{t} E_{\ell}^{t+1}\right| \leq 1$ for all $t$. The goal of the pursuit game is to have all $M$ evaders eventually captured.

Thanks to Proposition 10, the obvious necessary condition for capture is that the Boundedness Condition is satisfied for each $E_{\ell}$ with respect to the entire collection of pursuers $\left\{P_{j}\right\}$. Thanks to Theorem 17, the obvious sufficient condition for capture is that there is a partition of the pursuers, $\left\{P_{\ell, i}\right\}$ for $i=1 \ldots M_{\ell}$, such that for each $\ell$, the collection $\left(E_{\ell},\left\{P_{\ell, i}\right\}\right)$ satisfies the Boundedness Condition on $\mathcal{D}$. The obvious strategy in this case is to play games in parallel.

In the case of multiple evaders, more complex strategies of pursuers' trading 'ownership' of an evader are possible. This remains an important and interesting challenge.

Remark 27. Information constraints. Similar network-theoretic issues surround issues of communication and exchange of information between players. The necessary and sufficient conditions of this paper have the stringent assumptions of perfect evader location and domain information, as well as initial all-to-all communication between pursuers (to initialize centers $O_{j}$ ). Relaxing these assumptions generates a number of interesting challenges.

For example, assume that the pursuers know only an approximate evader location, encoded as a compact convex set $\mathcal{E}$ (cf. [30]). Assume a monotonicity condition which says that the set $\mathcal{E}^{t+1}$ is a subset of the translation of $\mathcal{E}^{t}$ by a vector of at most unit length. (That is, uncertainty of evader location can decrease but cannot increase.) Then it is perhaps possible to reprove the Main Theorem by, for example, having each pursuer chase after the point of $\mathcal{E}$ closest to the pursuer's center (this is well-defined thanks to convexity).

Other scenarios for uncertain information include those in which the pursuers do not admit an initial all-to-all communication round, but rather communicate with pursuers which are sufficiently close. Far-off pursuers cannot be reached. This and similar problems touch on many ideas currently in play in the control theory literature on distributed consensus with limited/faulty communication [35]. 
Remark 28. Other noncooperative pursuit games. There are numerous examples of pursuit-evasion games beyond the Lion $\mathcal{E}$ Man setting: see $[12,25]$ for an overview. We mention in particular the case considered by Isaacs [12] in which the evader's goal is to reach a specified subset of the domain. More recent entries in the literature consider pursuit games in which capture means not physical coincidence, but rather visibility - the pursuer wins when there is a line-of-sight to the evader. For results in this genre, see $[34,9]$. More recently, much attention has been paid to probabilistic techniques in pursuit games: see $[13,14,37]$.

Stepping back from the game-theoretic perspective, one can consider a pursuit-evasion game as a form of cooperative consensus problem, where a "swarm" of pursuers attempts to reach positional consensus with an evasive "leader." Consensus problems have received a great deal of attention recently from the control-theory community, with motivation from biologically observed swarming phenomena. Several authors $[5,35,23]$ have given decentralized algorithms for reaching consensus in a variety of contexts.

\section{REFERENCES}

[1] S. Alexander, R. Bishop, and R. Ghrist, "Pursuit and evasion on non-convex domains of arbitrary dimensions," Proc. Robotics, Systems and Science, 2006.

[2] L. Alonso, A. Goldstein, and E. Reingold, "'Lion and man': upper and lower bounds," ORSA J. Comput. 4(4), 1992, 447-452.

[3] P. Bouguer, "Sur les lignes courbes qui sont propres à former les voûtes en dôme," Mémoires de l'Académie de Paris, 1732, 149-164.

[4] A. Chikrii and P. Prokopovich, "Simple pursuit of one evader by a group," Cybernetics E Sys. Analysis, 28:3, 1992, 438-444. Translated from Kibernetika i Sistemnyi Analiz, 3, 1992, 131-137.

[5] J. Cortés and F. Bullo, "Coordination and geometric optimization via distributed dynamical systems," SIAM J. Control E Optimization, 44:5, 2005, 1543-1574.

[6] H. Croft, "'Lion and Man': A Postscript." J. London Math. Soc. 39, 1964, 385-390.

[7] L. Danzer, B. Grünbaum, V. Klee, "Helly's theorem and its relatives," Proc. Sympos. Pure Math. Vol. VII, Amer. Math. Soc., 1963, 101-180.

[8] J. Flynn, “Lion and man: the general case," SIAM J. Control, 12, 1974, 581-597.

[9] L. Guibas, J.-C. Latombe, S. LaValle, D. Lin, and R. Motwani, “A visibility-based pursuit-evasion problem," Inter. J. Comput. Geom. E Applications 9(4-5), 1999, 471-.

[10] N. Hovakimyan and A. Melikyan, "Geometry of pursuit-evasion on second order rotation surfaces," Dynamics E Control 10(3) 2000, 297-312.

[11] G. Ibragimov, “On a game of optimal pursuit of one evader by several pursuers," Prikl. Mat. Mekh. 62(2), 1998, 199-205; transl. in J. Appl. Math. Mech. 62(2), 1998, 187-192.

[12] R. Isaacs, Differential Games, Wiley Press, NY, 1965.

[13] V. Isler, S. Kannan, and S. Khanna, "Locating and capturing an evader in a polygonal environment," in Proc. Workshop Alg. Foundations of Robotics, 2004.

[14] V. Isler, D. Sun. and S. Sastry, "Roadmap based pursuit-evasion and collision avoidance," in Proc. Robotics, Systems, \& Science, 2005.

[15] R. Ivanov, "Theorem on the alternative in simple pursuit-evasion and optimality on a half space," Serdica 10(4), 1984, 397-411.

[16] V. Jankovic, “About a Man and Lions," Mat. Vesnik 2, 1978, 359-361.

[17] S. Kopparty and C. Ravishankar, "A framework for pursuit-evasion games in $\mathbb{R}^{n}$," Information Proc. Lett., 96, 2005, 114-122. 
[18] A. Kovshov, "The simple pursuit by a few objects on the multidimensional sphere," Game Theory $\mathcal{E}$ Applications II, L. Petrosjan and V. Mazalov, eds., Nova Science Publ., 1996, 27-36.

[19] N.-M. Lê, "On determining Mathematical Games optimal strategies in pursuit games in the plane," Theoretical Computer Science, 197, 1998, 203-234.

[20] J. Lewin, "The lion and man problem revisited," J. Optim. Theory Appl., 49(3), 1986, 411-430.

[21] J. Littlewood, A Mathematician's Miscellany, Methuen \& Co., London 1953. Revised edition published as Littlewood's Miscellany, Cambridge University Press, 1986.

[22] A. Melikyan, Generalized Characteristics of First Order PDEs, Birkhauser, 1998.

[23] L. Moreau, "Stability of multiagent systems with time-dependent communication links," IEEE Trans. Aut. Control, 50:2, 2005, 169-182.

[24] F. Morley, "A curve of pursuit," Amer. Math. Monthly, 28(2), 1921, 54-61.

[25] P. Nahin, Chases and Escapes: The Mathematics of Pursuit and Evasion, Princeton Press, 2007.

[26] T. Parsons, "Pursuit evasion in a graph," in Theory \& Application of Graphs, Y. Alavi and D. Lick, eds., Springer-Verlag, 1976, 426-441.

[27] J. Reif and S. Tate, "Continuous alternation: The complexity of pursuit in continuous domains," Algorithmica, 10, 1993, 157-181.

[28] B. Rikhsiev, "Optimality of pursuit time in an $n$-person differential game with simple motion," Izv. Akad. Nauk UzSSR Ser. Fiz.-Mat. Nauk 4, 1984, 37-39.

[29] R. Rockafellar, Convex Analysis, Princeton Univ. Press, Princeton, 1970.

[30] G. Rote, "Pursuit-evasion with imprecise target location," Proc. 14th ACM-SIAM Symposium on Discrete Algorithms, 2003, 747-753.

[31] N. Satimov and A. Kuchkarov, "Deviation from encounter with several pursuers on a surface." Uzbek. Mat. Zh. 1, 2001, 51-55.

[32] N. Satimov and A. Kuchkarov, "On the solution of a model differential pursuit-evasion game on a sphere." Uzbek. Mat. Zh. 1, 2000, 45-50.

[33] J. Sgall, "Solution of David Gale's lion and man problem," Theor. Comp. Sci. 259, 2001, 663-670

[34] I. Suzuki and M. Yamashita, "Searching for a mobile intruder in a polygonal region," SIAM J. Comput., 21(5), 1992, 863-888.

[35] H. Tanner, A. Jadbabaie, and G. J. Pappas, "Flocking in fixed and switching networks," IEEE Trans. Aut. Control, 52:5, 2007, 863-868.

[36] D. Vagin and N. Petrov, "The problem of the pursuit of a group of rigidly coordinated evaders," Izv. Akad. Nauk Teor. Sist. Upr. 5, 2001, 75-79.

[37] R. Vidal O. Shakernia, H. Kim, D. Shim, and S. Sastry, "Probabilistic pursuit-evasion games: theory, implementation, and experimental evaluation," IEEE Trans. Robotics $\mathcal{E}$ Aut. 18, 2002, 662-669.

[38] H. Wu, "The spherical images of convex hypersurfaces," J. Differential Geometry, 9, 1974, 279-290.

DEPARTMENT OF MATHEMATICS, UNIVERSITY OF ILLINOIS, URBANA IL, 61801

E-mail address: sba@math.uiuc.edu

DePARTMENT OF MATHEMATICS, UNIVERSity OF ILLINOIS, URbANA IL, 61801

E-mail address: bishop@math.uiuc.edu

DEPARTMENT OF MATHEMATICS AND COORDINATED SCIENCE LABORATORY, UNIVERSITY OF ILLINOIS, URBANA IL, 61801

E-mail address: ghrist@math.uiuc.edu 